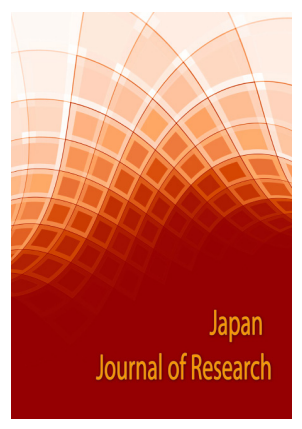

Correspondence

Yu Koryak

SSC, Institute of Biomedical Problems RAS, 76-A Khoroshevskoye Shosse, 123007,

Moscow, Russia

E-mail:yurikoryak@mail.ru

- Received Date: 25 May 2021

- Accepted Date: 08 June 2021

- Publication Date: 14 June 2021

\section{Copyright}

(c) 2021 Science Excel. This is an openaccess article distributed under the terms of the Creative Commons Attribution 4.0 International license.

\title{
Kinetics of muscular function and architecture changes during of chronic low-frequency electrical stimulation
}

\author{
Yu Koryak \\ SSC, Institute of Biomedical Problems RAS, 76-A Khoroshevskoye Shosse, 123007, Moscow, Russia
}

\begin{abstract}
The effect of a 7-day "dry" water immersion (DI) with countermeasures (neuromuscular electrical stimulation - NMES) on the function and architecture of the human triceps surae muscle was studied in six healthy young men. During DI, subjects performed NMES muscle groups of both lower extremities every day. Internal architecture was measured in vivo by use of B-mode ultrasonography. The ankle was positioned at $-15^{\circ}$, and $0,+15^{\circ}$, and $+30^{\circ}$ plantarflexion. At each position, longitudinal ultrasonic images of the medial and lateral gastrocnemius and soleus muscles were obtained while the subject was relaxed and performed $50 \%$ maximal voluntary isometric contraction.
\end{abstract}

\section{Introduction}

Gravitational loading appears to be necessary for the maintenance of human lower limb skeletal muscle size and force [1]. Studies simulating microgravity have shown that exercise countermeasures can attenuate, but not completely prevent the loss of muscle mass and force $[2,3]$. The muscle groups most affected by exposure to microgravity appear to be the antigravity extensors of the knee and ankle [4]. Among these, the plantarflexors seem to be the most affected [4], likely due to their greater mechanical loading under normal gravitational conditions. Most notable after exposure to microgravity is a disproportionate loss of force as compared to that of muscle size [4], indicating that factors other than atrophy contribute to muscle weakness. The internal architecture of a muscle is an important determinant of its functional characteristics. There is a paucity of studies on the effects of disuse [5] or simulated microgravity [1,6] or real microgravity [7] on muscle architecture. The purpose of the present study was to investigate the internal architecture of the triceps surae [medial (MG) and lateral (LG) and soleus (SOL) muscles] in relation to the functional characteristics of the plantarflexors after 7 days of «dry» water immersion (DI) with exercise countermeasures [neuromuscular electrical stimulation (NMES)].

Materials and methods

Participants

We recruited six young men-volunteers (aged $22.8 \pm 0.8 \mathrm{yr}, 1.84 \pm 0.1 \mathrm{~m}$, and $79.3 \pm$ $4.2 \mathrm{~kg}$, mean \pm s.e.m). Selection of subjects was based on a screening evaluation that consisted of a detailed medical history, complete blood count, urine analysis, resting and cycle ergometer electrocardiogram, and a panel of blood chemistry analysis, which included fasting blood glucose, blood urea nitrogen, creatinine, lactic dehydrogenase, bilirubin, uric acid, and cholesterol, as well as an evaluation of their physical state using a bicycle ergometer stresstest. Continuous, gradually increasing work in the bicycle ergometer at a constant pedaling rate of $60 \mathrm{rpm}$ over $3 \mathrm{~min}$ with initial load of $50 \mathrm{~W}$ was specified; the load of subsequent "steps" was increased by $25 \mathrm{~W}$; achievement of submaximal heart rate was the criterion for work termination.

All of the subjects were evaluated clinically and considered to be in good physical condition. No subject was taking medication at the time of the study, and all subjects were nonsmokers and with no neurological disorders participated. All subjects gave written informed consent in accordance with the Declaration of Helsinki. The experimental protocol was approved by the Institute of Bio-Medical Problems Ethic Committee.

\section{"Dry" water immersion}

To simulate microgravity the DI model has been used [8]. This model seems to be a useful method for ground based investigations. As it has been shown in prevision studies [9] a close similarity exists between the effects of short time real microgravity and immersion. However, the dimension of these changes is different. The alterations during and after immersion are more marked than those of equal duration spaceflights.

Briefly, the subject was horizontally positioned 
(a angle which make the body and horizontal line, e.g. $5^{\circ}$ headup position) in a special bath in a "suspended" state (the law of Archimedes), on special water-proof highly elastic fabric film, which acted only as an insulation between the skin and the water. There was no bed to support the subject's body. The folds of the water-proof material came together with water along the midline of the subject's body. Water temperature was constant $\left(33.4^{\circ}\right.$ C) and maintained automatically at this level throughout the experiment. The exposure duration was 7-day and the subjects were kept under medical observation throughout the exposure. During the 7-day DI, the subjects remained in the experimental (horizontal) position continuously for all activities including voiding, and eating.

\section{Neuromuscular electrical stimulation}

NMES is applied to 4 muscle groups of both lower extremities. "Dry" electrodes (Ltd. "Axelgaard», USA) are placed on the skin above the quadriceps femoris muscles, the hamstrings, the tibialis anterior, the peroneal, and the triceps surae muscles. The synchronous stimulation of antagonistic muscle groups prevents unwanted joint movements. The NMES-training is performed during 3 hours per day with $1 \mathrm{~s}$ " on " and $2 \mathrm{~s}$ " off» trains at intensity levels of $20-30 \%$ of maximum tetanic force and a frequency of $25 \mathrm{~Hz}$. The electrical stimulus was provided by the «STIMUL LF-1» stimulator (Russia). The technical equipment consists of electrode trousers carrying stimulation electrodes for the 12-channels, and 2 interconnected 6-channel stimulators caned on a belt. During DI, subjects executed a NMES-training during 3 hours per day with $1 \mathrm{~s}$ " on " and 2 s «off» with a frequency of $25 \mathrm{~Hz}$ and amplitude of stimulus from 0 up to $45 \mathrm{~V}$ for training. Used biphasic rectangular by $1 \mathrm{~ms}$ pulse width. After initialization procedure, the system begins automatic training. NMES-training of muscles of the examinee was carried out directly in a bath. The intensity level stimulation is determined by a threshold of bearableness of subjects.

NMES-training continued for six days, during which daily five days on end (from Monday to Friday inclusive) including one day of rest (Saturday). Duration of NMES-training was 3 hours/ day. Each subjects instructed "increase amplitude stimulation pulse during training".

Measurement of Maximal Voluntary Contraction Torque.

All subjects were instructed to abstain from food for 2 hours before testing, from caffeine for 4 hours before testing, and from exercise for 12 hours before testing. Before isokinetic testing was conducted, subjects pedaled a cycle ergometer at a workload of $25-50 \mathrm{~W}$ at a cadence of $60-80 \mathrm{rpm}$ for $5 \mathrm{~min}$. Standard jointspecific warm-up procedures were followed and consisted of five submaximal repetitions and two to three maximal repetitions. After the warm-up, subjects rested at least $2 \mathrm{~min}$. Strength tests were performed such that subjects exerted a maximal effort in only one direction for each set of repetitions.

After a full warm-up, isometric ankle extension torque of the subjects' right legs was tested at joint angular velocities of $0 \% \mathrm{~s}-1$ using a Isokinetic Dynamometer (Biodex System $4 \mathrm{PRO}^{\mathrm{mm}}$, Biodex Medical Systems, Shirley, New York, USA). Participants were seated upright in the Biodex dynamometer chair with their trunk positioned and secured to the seatback with waist and shoulder belts to ensure consistent positioning and minimal movement. The hips were positioned to $90^{\circ}$ with the thigh and the knee angles at $45^{\circ}$ flexed and the ankle was in the $90^{\circ}$ joint angle were taken to determine the plantarflexion torque. The lateral malleolus of the right foot was aligned with the axis of rotation on the Biodex dynamometer (Biodex Medical Systems, N-Y). The foot was fastened to the footplate with inelastic straps that were firmly secured behind and on the underside of the footplate to prevent heel lift. When heel lift occurred or torque did not return to baseline protocol was stopped and repeated after 3-5- min rest. Subjects performed three sets of four repetitions of maximal isometric ankle extension at an angular velocity of $0 \% \mathrm{~s}-1$ with a 2-min resting period between contractions unless the third trial exceeded one of the two first ones by more than $10 \%$. In that case an additional trial was performed. The participants were instructed to grip the side handles to help stabilize the trunk.

Each subject was instructed to exert maximal effort in only one direction and in every movement when performing a test; no verbal instruction was provided during testing. Two min rest separated the sets. Isometric of peak torque at $0 \% \mathrm{~s}-1$ (maximal voluntary contraction - MVC) were recorded for each subject. The contractile properties of muscles were determined 2 days before the start of DI and 1 day after the end of DI.

\section{Measurement of Muscle Architecture}

Subjects performed a series of isometric plantarflexion contractions on an isokinetic dynamometer Biodex (Biodex Multi-Joint Systems, Shirley, N-Y, USA) at ankle angles of $0^{\circ}$ (neutral ankle position: the footplate of the dynamometer perpendicular to the longitudinal axis of the tibia). Before the strength measurements, muscle thickness, pennation angles and fascicle length of the right TS muscle were measured in vivo by B-mode ultrasonography (SonoSite MicroMaxx (SonoSite MicroMaxx, USA). All measurements were carried out with the knee joint flexed at $90^{\circ}$. To perform TS muscle ultrasound, a participant's foot was relatively rigidly fixed to a special platform, which allowed the ankle angle to be set at $-15^{\circ \circ}$ (plantar flexion), $0^{\circ \circ}$ (neutral anatomical position), $+30^{\circ \circ}$ (plantar extension). The MG, and LG, and SOL muscle architecture was assessed in vivo at rest and its change before and after 3 weeks DI, in the sagittal plane using B-mode ultrasonography, a linear $7.5-\mathrm{MHz}$ electronic transducer with a $60-\mathrm{mm}$ field of view at the proximal levels $30 \%$ of the distance between the popliteal crease and the center of the lateral malleolus [10]. The linear array probe was coated with water-soluble transmission gel to provide acoustic contact without depressing the dermal surface. The ankle joint was fixed at $-15^{\circ}$ (plantar flexion), $0^{\circ}$ (a neutral position), $+30^{\circ}$ (plantar extension). The knee joint was positioned at $0^{\circ}$ (full extension). Ultrasound images were captured at the required time point and stored for subsequent analysis. Before measurements, the subjects were laid on a couch for $20 \mathrm{~min}$ to allow osmotic fluids to shift [11]. During measurements, the subjects were laid supine with the knees fully extended and muscles relaxed. The MG, and LG, and SOL muscle fibre fascicle length and pennation angle (defined as the angle of insertion of fascicles into the deep aponeurosis) were measured using digitizing software (Image J, $1.47 \mathrm{v}$, National Institute of Health, Maryland, USA) before and after DI. The fascicle pennation angle $(\Theta f)$ was measured from the angles between the echo of the deep aponeurosis of each muscle and interspaces among the fascicles of that muscle [12]. The length of fascicles (Lf) across the deep and superficial aponeurosis was measured as a straight line [12]. This has been recently reported as a valid method of fascicle length estimation [13]. Images were collected and analysed by the same investigator.

After performance in the passive condition, the subject was encouraged to perform maximal voluntary isometric plantar flexion (active condition), and torque output was recorded. Each subject was then asked to maintain contractions for at least 2-3s at $50 \%$ of MVC at the neutral ankle position ( $0 \mathrm{deg})$. Subjects 
were given visual feedback of the target and elicited force on a computer screen. Shorter fascicle lengths and steeper fascicle angles in the active compared with the passive condition show internal shortening of fascicles by contraction. From these parameters, the $\Delta L$ muscle was estimated by the following equation:

\section{$\Delta L$ muscle $=L r \cdot \cos \Theta \mathrm{r}-L f \cdot \cos \Theta \mathrm{f}$, where}

$L r$ and $L f$ are fascicle lengths in rest (passive) and active conditions (50\% MVC);

$\Theta r$ and $\Theta f$ are fascicle angles in rest (passive) and active conditions, respectively.

\section{Statistics}

Data are presented as the mean values \pm standard error of the mean (SE). Differences in pennation angles, fibre lengths and thicknesses between rest and 50\% MVC and between different ankle angles were tested using two-way analysis of variance tests. Tukey's test was used to determine significant difference between mean values. One-way analysis of variance (ANOVA) was used for comparison of muscle thickness, pennation angles, and fibre lengths. A level of $p<0.05$ was selected to indicate statistical significance.

\section{Results}

\section{Changes in maximal muscle strength}

After the 7-day DI with application by NMES-training, maximal plantar flexion torque by three subjects has increased on the average by $11.3 \%(150 \pm 17.3$ vs $167 \pm 6.7 \mathrm{H})$ and at one has decreased for $9.6 \%$ (155 vs $140 \mathrm{H})$.

\section{Architectural characteristics}

After DI, in the passive condition, Lf in the MG, and LG, and SOL has decreased for 12 (from $32 \pm 2$ to $28 \pm 1 \mathrm{~mm}$ ), 13 (from $36 \pm 2$ to $31 \pm 2 \mathrm{~mm}$ ), and $13 \%$ (from $36 \pm 3$ to $32 \pm 2 \mathrm{~mm}$ ) but in the active condition by 18 (from $26 \pm 3$ to $22 \pm 2 \mathrm{~mm}$ ), 22 (from $36 \pm 3$ to $28 \pm 2 \mathrm{~mm}$ ), and $21 \%$ (from $32 \pm 2$ to $26 \pm 2 \mathrm{~mm}$ ), respectively. The $\theta \mathrm{f}$, in the passive condition, was decreased by 22,20 and $16 \%$; but in the active condition by 17,22 and $17 \%$, respectively.

Shorter fascicle lengths and steeper fascicle angles in the active compared with the passive condition show internal shortening of fascicles by contraction. Before DI $\Delta L$ muscle the MG has found $7.9 \mathrm{~mm}$ after has decreased and has made $7.8 \mathrm{~mm}$, and in Sol 5.9 $v s 5.6 \mathrm{~mm}$. Significant increase in $\Delta L$ muscle from 0.9 to $3.3 \mathrm{~mm}$ were found by LG.

\section{Discussion}

This study describes, for the first time, the architecture of the human TS [medial (MG) and lateral (LG) gastrocnemius and soleus (SOL) muscles] in vivo, both at rest and after 7 days of unloading with exercise countermeasures (NMES).

After 7 days of DI a considerable increased of MVC, was observed in the exercise $(+11 \%)$ groups whereas absence of preventive actions results in reduction in MVC more than on $30 \%$ [14-16]. Internal architecture of the GM, and LG, and SOL muscles was altered and this was only partially prevented by exercise countermeasures. Both fascicle length and pennation angle were reduced after DI with NMES, this strongly suggests a loss of both in-series and in-parallel sarcomeres, respectively. The functional consequence of the decreased fascicle length was a reduced shortening during contraction. The loss of in-series sarcomeres would mean that this is likely to have implications both on the force-length and force-velocity relationships of the muscle [17]. The observation of a smaller pennation angle during contraction after DI with NMES will partially compensate for the loss of force, because of a more efficient force transmission to the tendon. The reduced initial resting pennation angle probably, grows out reduction decreased tendon stiffness or of the muscle-tendon complex that finds confirmation in substantial growth $\Delta L$ muscle of LG (with 0.9 up to $3.3 \mathrm{~mm}$ after DI) during contraction. This observation is consistent with the findings of Kubo et al. [1]. A lower $\Theta f$ observed after a simulated microgravity [6] partly compensates for the loss of force because the force transmission to the tendon becomes more efficient in spite of the decreased stiffness of the muscle-tendon complex $[15,16]$.

The increase in the maximal voluntary torque after DI with NMES-training allows to assume, that NMES-training, apparently, promotes increase stream muscular afferentation [18] in conditions of his deficiency at gravitational unloading the muscular device caused long immersion that can promote also to the certain role in maintenance and normalization of activity of control systems by any movements (by a principle of a feedback). Tetanic electrical stimulation applied over human muscle generates contractions by depolarizing motor axons beneath the stimulating electrodes. However, the simultaneous depolarization of sensory axons can also contribute to the contractions by the synaptic recruitment of spinal motoneurons. Upon entering the spinal cord, the sensory volley recruits spinal motoneurons, leading to the development of central torque. This recruitment is consistent with the development of persistent inward currents in spinal motoneurons or interneurons $[19,20]$. Persistent inward currents lead to sustained depolarizations (plateau potentials), and it is becoming increasingly clear that they play an important role in regulating cell firing in normal $[21,22]$. Maximizing this central contribution may be beneficial for increased muscle force (by a principle of a feedback).

Conclusions, from the present results, follows, first, that the architecture different lead the triceps surae muscle considerably differs, reflecting, probably, their functional roles, second, various changes fibre length and pennation angle between different muscles, probably, are connected to distinctions in ability to develop force and elastic characteristics of sinews or muscle-tendon complex and, at last, in the third, NMEStraining has preventive an effect on stimulated muscles: in part reduces loss of force of reduction of the muscles, the caused long unloading. The received data, allow concluding, that use of NMES-trained renders the expressed preventive action, essentially reduces depth and rate of atrophic processes in muscles.

Present results suggest that the structural adaptations to immersion (unloading) likely to contribute to a reduced force loss. On this background used NMES training of muscles in conditions of unloading allows to increase contractile function.

\section{Acknowledgments}

The author is grateful to all volunteers of this study for their commitment and enthusiastic participation in the study, the medical and engineering staff for contribution to the study organization, and many unidentified colleagues for help in research.

\section{Grants}

The study is supported by Ministry of Education and Science of the Russian Federation Grant RFMEFI61317X0074.

\section{Disclosures}

No conflicts of interest, financial or otherwise, are declared by the author.

\section{References}

1. Kubo K, Akima H, Kouzaki M, et al. Changes in the elastic properties of tendon structures following 20 days bed-rest in humans. Eur J Appl Physiol. 2000;83(6):463-468. 
2. Kawakami Y, Muraoka Y, Kubo K, Suzuki Y, Fukunaga T. Changes in muscle size and architecture following 20 days of bed rest. J Gravit Physiol. 2000;7(3):53-59.

3. Koryak YU. Electrically evoked and voluntary properties of the human triceps surae muscle: effects of long-term spaceflights. Acta Physiol Pharmacol Bulg. 2001;26(1-2):21-27.

4. LeBlanc A, Gogia P, Schneider V, Krebs J, Schonfeld E, Evans $\mathrm{H}$. Calf muscle area and strength changes after five weeks of horizontal bed rest. Am J Sports Med. 1988;16(6):624-629.

5. Maganaris CN, Baltzopoulos V, Sargeant AJ. Changes in Achilles tendon moment arm from rest to maximum isometric plantarflexion: in vivo observations in man. J Physiol. 1998;510 ( Pt 3)(Pt 3):977-985.

6. Koryak YA, Kuzmina MM, Berezhinsky IV, Kovalenko VM. Long-term electromyostimulation training of muscles in a man in the conditions of mechanical unloading of the muscular apparatus and its influence on the architecture and function of the human triceps surae muscle. Sci J Fundamental Res. 2010;3:68-87.

7. Koryak YA. Architectural and functional specifics of the human triceps surae muscle in vivo and its adaptation to microgravity. J Appl Physiol (1985). 2019;126(4):880-893.

8. Shul'zhenko EB, Will-Williams IF. Possibility of carrying out prolonged water immersion by the method of "dry" submersion. Kosm Biol Aviakosm Med. 1976;10(2):82-84.

9. Kozlovskaia IB, Grigor'eva LS, Gevlich GI. Comparative analysis of the effect of weightlessness and its model on the velocitystrength properties and tonus of human skeletal muscles. Kosm Biol Aviakosm Med. 1984;18(6):22-26.

10. Kawakami Y, Ichinose Y, Fukunaga T. Architectural and functional features of human triceps surae muscles during contraction. J Appl Physiol (1985). 1998;85(2):398-404.

11. Berg HE, Tedner B, Tesch PA. Changes in lower limb muscle cross-sectional area and tissue fluid volume after transition from standing to supine. Acta Physiol Scand. 1993;148(4):379385.

12. Abe T, Kumagai K, Brechue WF. Fascicle length of leg muscles is greater in sprinters than distance runners. Med Sci Sports
Exerc. 2000;32(6):1125-1129.

13. Ando R, Taniguchi K, Saito A, Fujimiya M, Katayose M, Akima $\mathrm{H}$. Validity of fascicle length estimation in the vastus lateralis and vastus intermedius using ultrasonography. J Electromyogr Kinesiol. 2014;24(2):214-220.

14. Collins DF. Central contributions to contractions evoked by tetanic neuromuscular electrical stimulation. Exerc Sport Sci Rev. 2007;35(3):102-109.

15. Koryak YA. Influence of simulated microgravity on mechanical properties in the human triceps surae muscle in vivo. I: effect of 120 days of bed-rest without physical training on human muscle musculo-tendinous stiffness and contractile properties in young women. Eur J Appl Physiol. 2014;114(5):1025-1036.

16. Koryak Yu. Influence of simulated microgravity on mechanical properties in the human triceps surae muscle in vivo. II: Effect of 120 days of bed rest with physical training on human muscle musculo- tendinous stiffness and contractile properties in young women. Central Eur J Sport Med. 2015;11:125-143.

17. Narici MV, Maganaris CN. Plasticity of the muscle-tendon complex with disuse and aging [published correction appears in Exerc Sport Sci Rev. 2007 Oct;35(4):196]. Exerc Sport Sci Rev. 2007;35(3):126-134.

18. Gazenko OG, Grigoriev AI, Kozlovskaya IB. Mechanisms of acute and chronic effects of microgravity. Physiologist. 1987;30(1 Suppl):S1-S5.

19. Collins DF, Burke D, Gandevia SC. Large involuntary forces consistent with plateau-like behavior of human motoneurons. J Neurosci. 2001 Jun 1;21(11):4059-65.

20. Collins DF, Burke D, Gandevia SC. Sustained contractions produced by plateau-like behaviour in human motoneurones. J Physiol. 2002;538(Pt 1):289-301.

21. Collins DF, Gorassini M, Bennett D, Burke D, Gandevia SC. Recent evidence for plateau potentials in human motoneurones. Adv Exp Med Biol. 2002;508:227-235.

22. Heckmann CJ, Gorassini MA, Bennett DJ. Persistent inward currents in motoneuron dendrites: implications for motor output. Muscle Nerve. 2005;31(2):135-156. 